\title{
Virtual Healthcare Center for COVID-19 Patient Detection Based on Artificial Intelligence Approaches
}

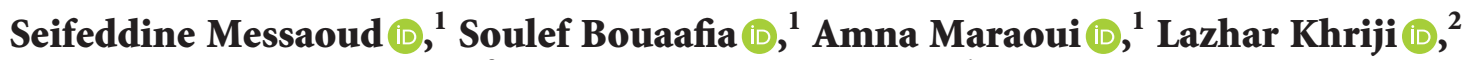 \\ Ahmed Chiheb Ammari $\mathbb{D}^{2}{ }^{2}$ and Mohsen Machhout $\mathbb{D}^{1}$ \\ ${ }^{1}$ Laboratory of Electronics and Microelectronics, Faculty of Sciences, University of Monastir, Monastir, Tunisia \\ ${ }^{2}$ Department of Electrical and Computer Engineering, College of Engineering, Sultan Qaboos University, Muscat, Oman \\ Correspondence should be addressed to Seifeddine Messaoud; seifeddine.messaoud@fsm.rnu.tn
}

Received 19 May 2021; Accepted 22 December 2021; Published 11 January 2022

Academic Editor: Mariam Arabi

Copyright (C) 2022 Seifeddine Messaoud et al. This is an open access article distributed under the Creative Commons Attribution License, which permits unrestricted use, distribution, and reproduction in any medium, provided the original work is properly cited.

\begin{abstract}
At the end of 2019, the infectious coronavirus disease (COVID-19) was reported for the first time in Wuhan, and, since then, it has become a public health issue in China and even worldwide. This pandemic has devastating effects on societies and economies around the world, and poor countries and continents are likely to face particularly serious and long-lasting damage, which could lead to large epidemic outbreaks because of the lack of financial and health resources. The increasing number of COVID-19 tests gives more information about the epidemic spread, and this can help contain the spread to avoid more infection. As COVID-19 keeps spreading, medical products, especially those needed to perform blood tests, will become scarce as a result of the high demand and insufficient supply and logistical means. However, technological tests based on deep learning techniques and medical images could be useful in fighting this pandemic. In this perspective, we propose a COVID-19 disease diagnosis (CDD) tool that implements a deep learning technique to provide automatic symptoms checking and COVID-19 detection. Our CDD scheme implements two main steps. First, the patient's symptoms are checked, and the infection probability is predicted. Then, based on the infection probability, the patient's lungs will be diagnosed by an automatic analysis of X-ray or computerized tomography (CT) images, and the presence of the infection will be accordingly confirmed or not. The numerical results prove the efficiency of the proposed scheme by achieving an accuracy value over $90 \%$ compared with the other schemes.
\end{abstract}

\section{Introduction}

The new coronavirus disease (COVID-19) appeared at the end of December 2019 in Wuhan city of China and has affected most of countries around the world $[1,2]$. COVID19 is caused by the novel severe acute respiratory syndrome coronavirus 2 (SARS-CoV-2) and represents the causative agent of a potentially fatal disease, making it a global public health concern. In this context, person-to-person COVID19 infection transmission has led to the isolation of patients who are subsequently administered a variety of treatments. In general, COVID-19 is an acutely resolved disease, but it can also be fatal, with a high fatality rate.

As of August 25, 2020, nearly 815,113 COVID-19 victims have died, while the total number of infected subjects was approximately $23,652,302$ cases. This is caused by SARSCOV-2, currently spread all over the world [3]. The outbreak began in Mainland China, with a geographic concentration in Wuhan City, Hubei. However, on February 26, 2020, the rate of increase in cases became higher in the rest of the world compared to that in China. Large outbreaks are occurring on a daily basis in Italy $(69,176$ cases), the United States (51,914 cases), and Iran (24,811 cases), and the geographic spread of the epidemic continues. The respiratory transmission of the disease from one person to another has caused the epidemic to spread rapidly, where the most common infection signs include respiratory issues, fever, cough, symptoms, and dyspnea.

Given the high number of infections and blockages at the hospital level, this disease can be mortal as a result of delayed 
detection and progressive respiratory failure [3]. Therefore, automatic and early COVID-19 diagnosis using advanced artificial intelligence (AI) techniques can aid countries in terms of rapid patient quarantining and disease monitoring. Neither chemicals nor enormous medical capabilities are required to perform the tests, and data analysis does not require long periods.

In this vein, deep learning, an important breakthrough in the AI field, has shown enormous potential for extracting tiny features in image analysis [4]. Because of the COVID-19 pandemic, some deep learning approaches have been proposed to detect patients infected with the coronavirus. Loey et al. developed a deep learning system based on a generative adversarial network (GAN) with deep transfer learning for coronavirus detection in chest X-ray images [5]. To recognize COVID-19, the authors of [6] proposed a transferlearning algorithm with convolutional neural networks using X-ray images. The authors of [7] proposed deep long short-term memory (LSTM) technique-based COVID-19 analysis that can recognize positive COVID-19 cases from both negative and healthy COVID-19 cases via cough and breath recordings on smartphones or wearable sensors. A deep learning technique-based early screening model was proposed by Xiaowei et al. [8] to detect COVID-19, Influenza, and viral pneumonia using computerized tomography (CT) images. The authors of [9] proposed fast algorithmbased deep learning to extract COVID-19 features to be used for diagnosis before pathogenic tests, which saves critical time. In addition, a deep learning-based model for detecting novel coronavirus pneumonia on high-resolution CT was suggested in [10]. Therefore, according to the development of intelligent techniques, future research directions may include more robust training strategies, for example, combining GAN with a genetic algorithm, incorporating edge and texture enhancement, reducing possible hallucination, coupling with explainable AI (XAI) modules, and exploring uncertainty measures in Bayesian deep attentive neural networks.

In summary, these deep learning tools-based models, which use either CT images or X-ray images, focus only on COVID-19 detection at the lung level, which is inconvenient for large populations as it requires scans of individual patients to detect the disease. In addition, the disease may be in the incubation period, which means it might be undetected by $\mathrm{X}$-rays and limited to the lung levels. To address this problem, the main contribution of this paper is to propose an innovative healthcare center to provide a COVID-19 disease diagnosis (CDD) framework based on deep learning techniques. However, our CDD framework considers both $\mathrm{CT}$ and X-ray images and provides a screening phase for the patient's symptoms before proceeding to the detection phase. The proposed framework implements two modules. First, a patient's symptoms checker (PSC) based on the regression technique is developed to provide automatic initial medical screening for patients. This module predicts how much a patient is likely to be infected based on their symptoms. Conforming to the infection probability results obtained, X-ray or CT images are taken. These images are then used by the second module, which performs deep learning-based COVID-19 automatic detection to confirm or infirm the presence of the infection. An application is developed to experiment with both modules at the cloud level. In this paper, the proposed novel framework is explained in detail, and experimental validations are performed.

The remainder of this paper is organized as follows. In Section 2, the related work to COVID-19, deep learning techniques, and medical images will be provided. Then Section 3 discusses the architecture of the proposed deep learning-based CDD tool. In Section 4, we describe the collection of the COVID-19 dataset of the proposed framework. Section 5 discusses the experimental results of the proposed scheme. Lastly, the conclusion of this paper is provided in Section 6.

\section{Related Work}

Unlike bacterial pneumonia, other types of lung infections are caused by viruses and are commonly called viral pneumonia. These viruses infect the lungs by blocking the oxygen flow, which can be life-threatening. COVID-19 is a recent example, defined as viral pneumonia caused by a new coronavirus that has quickly spread around the world and infected human life [11, 12]. In this context, SARS [13], COVID-19, and MERS [14] are studied as serious types of viral pneumonia which have spread continually to the pandemic level. Therefore, the development of an intelligent technique for viral pneumonia detection and infected cases recognition is recommended.

During the COVID-19 pandemic, viral nucleic acid detection using real-time polymerase chain reactions (RTPCR) is the standard diagnostic method $[15,16]$. Therefore, several hyperendemic nations are notable for providing sufficient RT-PCR tests for a million suspect cases in a short period. However, this method fails to recognize the new coronavirus before extracting the new virus's DNA sequence. Medical imaging is the most important method to help physicians assess disease development and take preventive measures as rapidly as possible. Clinically, chest radiography is the most widely used imaging modality in the patient's diagnosis of thoracic abnormalities given its effectiveness and significantly low cost [17]. Compared with a CT scan, an X-ray image cannot provide a 3D image of the human body's anatomy, but it is able to distinguish between viral pneumonia and nonviral pneumonia. In this context, a group of authors retrospectively studied the relationship between the chest CT results of examinations and clinical symptoms, laboratory tests, and other clinical factors for RTPCR-positive cases. They used a machine learning (ML) method-based systematic decision support system to predict the chest CT results for RT-PCR-positive pediatric patients. Their proposed system achieved an AUC of 0.84 with 0.82 accuracy and 0.84 sensitivity for predicting CT outcomes. Similarly, another recent study was presented for the automatic segmentation of high-intensity scar tissue and left atrium anatomy in the 3D LGE-CMR images of atrial fibrillation patients. For the segmentation of both the scar tissue and the left atrium, a method based on a multiview 
two-task (MVTT) recursive attention model was proposed, which consists of three subnetworks incorporating multiview learning, ConvLSTM, and an attention mechanism. The mean dice scores of $93 \%$ and $87 \%$ were obtained for LA anatomy and scar tissue, respectively.

Typically, radiography is a technique used to quantify the functional and structural consequences of chest disease, with the aim to provide high-resolution images of the disease progression. Several works have been carried out in this context. Pneumonia incidence detected via X-ray imaging was documented by the authors of [18] to be guided by subsequent vaccine treatment. In [19], the contributors described the most common lung abnormality patterns in COVID-19 using chest X-rays and affirmed that the medical community can rely on chest X-rays because of their wide availability and reduced problems. The authors of [20] have proven that common CT findings of peripheral distribution and bilateral involvement can also be seen on chest X-rays, showing the potential of chest X-rays as a powerful tool for COVID-19 recognition. Another experimental chest X-ray scoring system was proposed in [21] to be applied to hospitalized, infected patients with COVID-19.

Unlike these studies, we aim to screen for viral pneumonia and to develop a rapid and intelligent scheme based on deep learning techniques to differentiate infected patients with viral pneumonia from uninfected patients for the control and prevention of a possible epidemic.

Recent advancements in AI (deep learning) have made it possible to break into many long-standing medical image analysis tasks, such as monitoring, detecting, recognizing, and delineating pathological abnormalities. In the task of interpreting X-rays, deep convolutional nerve networks (DCNNs) have been established to diagnose and identify the most common and important chest diseases [22-25] and to distinguish between viral pneumonia and bacterial pneumonia $[26,27]$. In this context, many scientific researchers have used deep learning for the interpretation of radiographic images, with the aim of improving efficiency and reducing the burden on radiologists. The authors of [22] proposed a supervised framework for the classification and localization of fourteen common chest diseases. In [23], 121 dense convolutional neural network layers have been created to perform recognition tasks better than a radiologist. An awareness mechanism was proposed by the authors of [24] to help the model focus on the lesion area and thus further improve diagnosis performance. This is done using the correlation between class labels and pathological abnormality locations and by analyzing the feature maps learned by the classification branch. In addition, numerous attempts $[26,27]$ have been made to detect and differentiate between viral pneumonia and bacterial pneumonia based on DCNN classification models.

However, researchers have argued that many ML and deep learning-based models have major drawbacks because of flawed methodologies, underlying high biases in data collected from numerous public data repositories that have little verification opportunity, inadequate validation using external datasets that lack generalizability, and the insufficient documentation of publicly available datasets and codes for replicability. Thus, high-quality data, robust methodologies, demonstrations of strong validation using internal and external datasets and external models, and the availability and sufficient documentation of public datasets and codes are necessary to develop a trusted and dependable model that can provide consistent accuracy across seen and unseen data and contexts.

The main contribution of this paper is to propose an innovative healthcare center to provide a CDD framework based on deep learning techniques. However, the proposed CDD framework considers both CT and X-ray images and provides a screening phase for a patient's symptoms before proceeding to the detection phase.

\section{Proposed Healthcare Center Framework Based on Deep Learning Models}

Early warning and prediction is one of the most powerful technologies to fight the COVID-19 pandemic. In this context, the proposed healthcare center for CDD implements two phases as well as PSC and COVID-19 diagnosis. However, at the first stage, patients will undergo the symptoms checking phase with the aim to predict how much they are likely to be infected based on these symptoms. Then, conforming to the obtained infection probability results, X-ray or CT images at the lung level are taken. These images are then used by the second module, which performs deep learning-based COVID-19 automatic detection to confirm or infirm the presence of the infection. ML and deep learning techniques [28] are used in this contribution. The following sections will provide a detailed overview of the models that will then be implemented in the healthcare center, as shown in Figure 1.

3.1. Patient's Symptoms Checker (PSC) Model Based on ML Techniques. COVID-19 is mainly transmitted by droplets generated when an infected person coughs, sneezes, or exhales [29]. However, these droplets are too heavy to hang in the air and quickly fall to floors or surfaces. The infection can then be spread by breathing in the virus close to an infected patient or by touching a contaminated surface. In more severe cases, the infection can cause acute respiratory syndrome, septic shock, viral pneumonia, multiorgan failure, and death. In this context, we assume that patients will undergo symptoms checking for the first time to predict the possibility of COVID-19 infection, as illustrated in Figure 2. However, the proposed PSC module can predict infection probability based on the patient's symptoms and ML techniques.

Before introducing the main system based on the ML tools, we review some popular algorithms. Then, based on their performances, one of them will be selected to be the brain of the symptoms checker model. Among these commonly used algorithms in many domains, we choose the KNN, SVM, and logistic regression methods.

3.1.1. KNN Technique. The K-nearest neighbors (KNN) technique is a supervised ML method that can be used for both classification and regression predictive problems [30]. However, this is mainly used for the classification of 


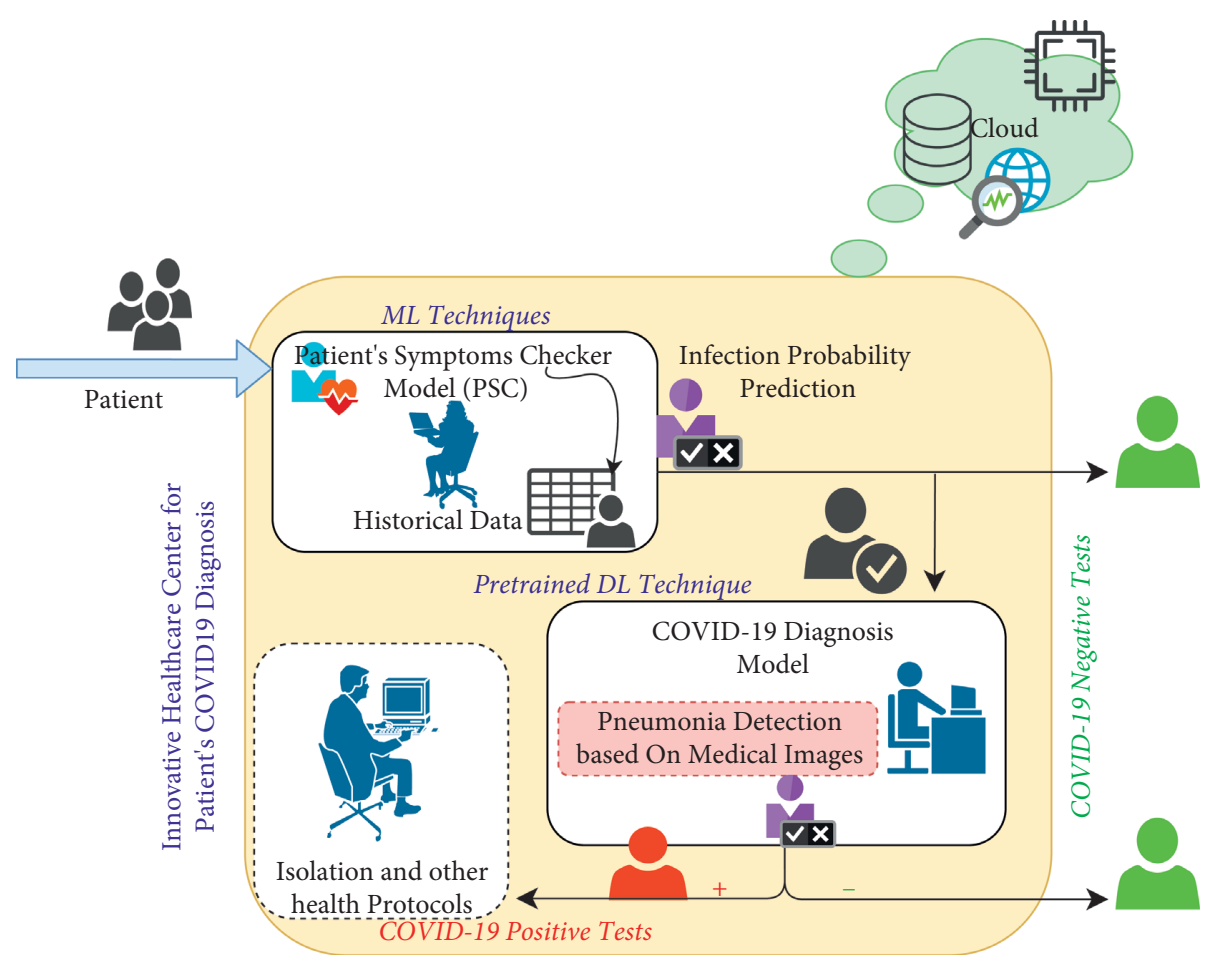

Figure 1: Proposed healthcare center for patient's COVID-19 diagnosis.

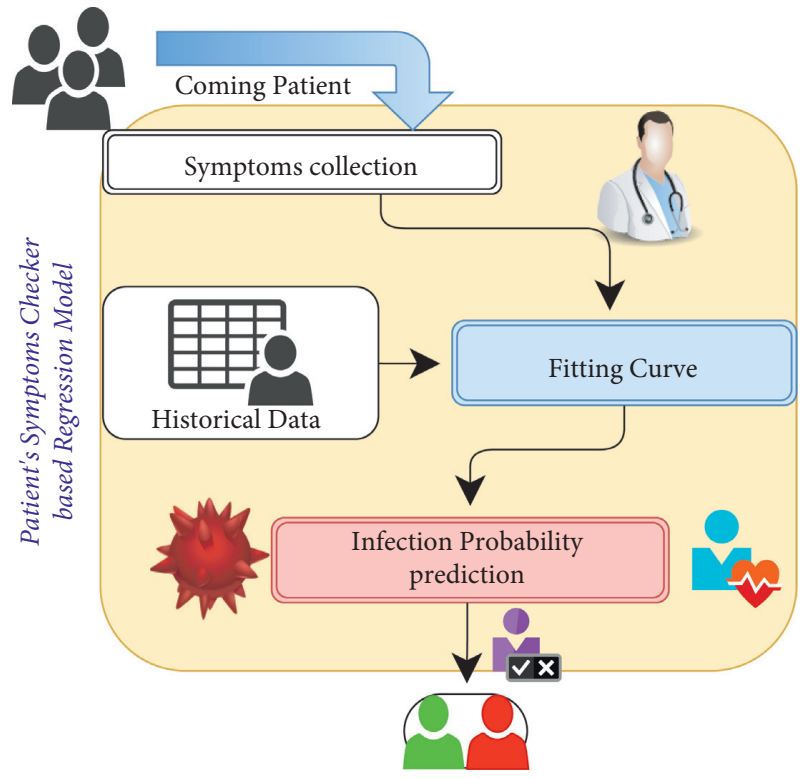

FIGURE 2: PSC based on regression model.

predictive problems in several areas. The two following properties would define KNN well:

(i) Lazy learning algorithm: KNN is a lazy learning algorithm because it does not have a specialized training phase and uses all the data for training during classification.

(ii) Nonparametric learning algorithm: KNN is also a nonparametric learning algorithm because it does not assume anything about the underlying data.
The KNN algorithm uses feature similarity to predict the values of new data points, which further means that the new data point will be assigned a value based on how closely it matches the points in the training set.

3.1.2. SVM Technique. Support vector machines (SVM) are powerful yet flexible supervised ML algorithms that are used for both classification and regression [31], but, generally, they are used in classification problems. SVM techniques have their unique ways of implementation as compared to other ML techniques. Lately, they have become extremely popular because of their ability to handle multiple continuous and categorical variables. An SVM model is basically a representation of different classes in a hyperplane in a multidimensional space. The hyperplane will be generated in an iterative manner via SVM so that the error can be minimized. The goal of SVM is to divide the datasets into classes to find a maximum marginal hyperplane $(\mathrm{MMH})$.

3.1.3. Logistic Regression Technique. Logistic regression is a supervised learning classification algorithm used to predict the probability of a target variable $[32,33]$. The nature of the target or dependent variable is dichotomous, which means there would be only two possible classes. Hence, the symptoms are considered as the input for the SPC model. In this context, let us denote by $X_{m}=\left\{x_{i}, \ldots, x_{n}\right\}$ the symptoms vector, and let $y_{m}$ be the predicted infection probability, where $m \in\{1, \ldots, M\}$ represents the data length ( $M$ patients, each having $i$ symptoms). The relationship between $X$ and $y$ is then modeled by the following equation, where $\beta$ 
represents the regression coefficient. Algorithm 1, summarizes the ML technique implementation.

$$
y_{i}=\frac{1}{1+\exp \left(-\left(\beta_{0}+\beta_{i} x_{i}+\cdots+\beta_{n} x_{n}\right)\right)} .
$$

3.2. COVID-19 Diagnosis Based on DL Model. In this section, the VGG19 model used for COVID-19 detections will be described first. Then the proposed COVID-19 diagnostic scheme based on the pretrained CNN model will be introduced. Subsequently, before proceeding to the learning phase and the experimental results, the used datasets in this simulation will be clarified.

3.2.1. Deep VGG Model. In this study, we explain in detail the selected pretrained VGG19 model and its structure. VGGNet was invented by Simonyan and Zisserman [34] at the University of Oxford Robotics. This deep network architecture has two versions with different depths and layers, namely, VGG16 and VGG19. In this work, VGG19 was used to achieve the clinical purpose of the COVID-19 model. Table 1 summarizes the VGG19 layers and parameters. The VGG19 architecture consists of five blocks of convolutional layers; each block is followed by a pooling layer and three fully connected layers at the end. At first, the input RGB image to the network is of the size of $224 \times 224 \times 3$, which passes through five convolutional layers with a kernel size of $3 \times 3$ and a stride of one. After the convolutional layers, the maxpooling layers are performed over a $2 \times 2$ kernel size with a stride of two. In the end, the last pooling layer is followed by the flattening layer and three fully connected layers for COVID-19 detection.

3.2.2. COVID-19 Detection Based on VGG19 Model. To detect COVID-19 from non-COVID-19 cases, we proposed a deep learning method in this study. Figure 3 describes the proposed method based on the deep CNN model; in particular, we have chosen VGG19. As shown in Figure 3, we developed a deep CNN-based VGG19 model for COVID-19 detection tasks (COVID-19 and non-COVID19 classes) using medical images. In the first stage, the medical images collected in the dataset can be processed later in the deep learning pipeline, loaded for a fixed size of $224 \times 224$. Then, to denote the positive case (COVID-19) or "not" for each subject in the dataset, we applied one-hot encoding on the labels of image data. In the second stage (the training phase), the preprocessed dataset will be divided into $80 \%$ for constructing equal training and validation sets and $20 \%$ for the testing phase. The selection of training data for the deep classifier will be a random subsample. Hence, to measure the performance of the validation set, we apply the evaluation metrics illustrated in the next section. In the final stage, the categorization of all images, classified into two categories-positive COVID-19 or non-COVID-19-is performed by transmitting the testing data.

\section{Dataset Collection}

4.1. Symptoms Dataset. To prevent the spread of the coronavirus and possibly help treat the disease before it gets worse, we have proposed an early detection method for COVID-19 by checking symptoms. In fact, a wide range of symptoms, ranging from mild to serious, appear within two to fourteen days of the virus infecting people with COVID-19. Among these symptoms, we can cite the following: shortness of breath or difficulty breathing, repeated shaking with chills, cough, fever, muscle pain, chills, sore throat, new loss of taste or smell, and headache [35].

In this context, for the automatic symptoms checker module, we used a public day-level information dataset on COVID-19 cases [35]. The dataset used in this model to train ML techniques consisted of 270 patients, each with their ID, gender, and age and the discussed symptoms. Each subject in the dataset, based on their symptoms, is classified as 0 to denote a normal case or 1 to denote an infected case. Table 2 further clarifies the dataset.

4.2. COVID-19 Dataset. The proposed deep learning model was trained and tested based on two public, open datasets of X-ray and CT images. Therefore, the first dataset consists of Computed Tomography (CT) images of normal patients and patients infected with COVID-19, which are taken from the GitHub repository [36]. From [37, 38], we selected an X-ray dataset containing normal patients and COVID19 patients.

In many countries, hospitals do not include both $\mathrm{CT}$ and $\mathrm{X}$-ray machines. In this case, the proposed CDD framework can support medical images diversity and can deal with both $\mathrm{CT}$ image and X-ray images. On the other hand, DNN requires a large database in the training phase to achieve a high testing accuracy level and solid model that can correctly do the prediction. However, we have combined these two datasets as shown in Table 3.

The proposed deep learning model was trained and tested based on two public open datasets of X-ray and CT images. Therefore, the first dataset consists of the CT images of normal patients and patients infected with COVID-19, which are taken from the GitHub repository [36]. From $[37,38]$, we selected an X-ray dataset containing normal patients and COVID-19 patients.

In many countries, hospitals do not have both $\mathrm{CT}$ and $\mathrm{X}$-ray machines. In this case, the proposed CDD framework can support medical image diversity and can deal with both $\mathrm{CT}$ and X-ray images. On the other hand, DNN requires a large database in the training phase to achieve a high testing accuracy level and a solid model that can correctly do the prediction. As such, we have combined these two datasets, as shown in Table 3.

Our main goal here is to achieve good results in detecting COVID-19 cases. In this work, the datasets are gathered into two subsets, $80 \%$ for training and $20 \%$ for testing, including normal and COVID-19 cases. Figure 4 shows the CT and $\mathrm{X}$-ray images. 
Input: Historical Symptoms Data $D=\left\{X_{m}, y_{m}\right\}$

Output: Infection probability $y_{m}^{*}$

(1) BEGIN

(2) Initial step: Data collection (save any input patient's data in the dataset

(3) Step 1: apply SVM technique to the input data.

(4) $\quad$ Fit $(x, y)$ : curve fitting

(5) Compute accuracy score

(6) Compute accuracy precision

(7) Compute accuracy recall

(8) Compute accuracy F1-score

(9) Compute accuracy support

(10) Step 2: apply KNN technique to the input data.

(11) $\quad \operatorname{Fit}(x, y)$ : curve fitting

(12) Compute accuracy score

(13) Compute accuracy precision

(14) Compute accuracy recall

(15) Compute accuracy F1-score

(16) Compute accuracy support

(17) Step 3: apply Logistic function to the input data.

(18) $\quad P_{\beta}\left(y_{m}=1 \mid X_{m}\right)=1 /\left(1+e^{-\left(\beta_{\text {in }} X_{\text {in }}\right)}\right)$

(19) Learning (curve fitting): find optimal $\beta^{*}$ parameters that minimize the objective function $J(\beta)$

(20) $\beta^{*}=\arg \min J(\beta)$, where $J(\beta)=-\log \left(P_{\beta}(y \mid X)\right)$

(21) Predict the infection probability $y_{m}^{*}=\arg \min \left(p_{\beta *}\left(y_{m} \mid X_{m}\right)\right)$

(22) $\quad$ Fit $(x, y)$ : curve fitting

(23) Compute accuracy score

(24) Compute accuracy precision

(25) Compute accuracy recall

(26) Compute accuracy F1-score

(27) Compute accuracy support

(28) End

Algorithm 1: ML techniques for patient's symptoms checker.

TABLE 1: VGG19 model for the proposed scheme.

\begin{tabular}{|c|c|c|}
\hline Layer (type) & Output shape & Parameters number \\
\hline Input_1 (inputLayer) & (None, 224, 224, 3) & 0 \\
\hline Block1_conv1 (conv2D) & (None, 224, 224, 64) & 1792 \\
\hline Block1_conv2 (conv2D) & (None, $224,224,64$ ) & 36928 \\
\hline Block1_pool (maxPooling2D) & (None, 112, 112, 64) & 0 \\
\hline Block2_conv1 (conv2D) & (None, $112,112,128)$ & 73856 \\
\hline Block2_conv2 (conv2D) & (None, $112,112,128$ ) & 147584 \\
\hline Block2_pool (maxPooling2D) & (None, $56,56,128)$ & 0 \\
\hline Block3_conv1 (conv2D) & (None, $56,56,256)$ & 295168 \\
\hline Block3_conv2 (conv2D) & (None, $56,56,256)$ & 590080 \\
\hline Block3_conv3 (conv2D) & (None, $56,56,256)$ & 590080 \\
\hline Block3_conv4 (conv2D) & (None, $56,56,256)$ & 590080 \\
\hline Block3_pool (maxPooling2D) & (None, $28,28,256)$ & 0 \\
\hline Block4_conv1 (conv2D) & (None, $28,28,512$ ) & 1180160 \\
\hline Block4_conv2 (conv2D) & (None, $28,28,512$ ) & 2359808 \\
\hline Block4_conv3 (conv2D) & (None, $28,28,512$ ) & 2359808 \\
\hline Block4_conv4 (conv2D) & (None, $28,28,512$ ) & 2359808 \\
\hline Block4_pool (maxPooling2D) & (None, $14,14,512$ ) & 0 \\
\hline Block5_conv1 (conv2D) & (None, $14,14,512$ ) & 2359808 \\
\hline Block5_conv2 (conv2D) & (None, $14,14,512$ ) & 2359808 \\
\hline Block5_conv3 (conv2D) & (None, $14,14,512)$ & 2359808 \\
\hline Block5_conv4 (conv2D) & (None, $14,14,512$ ) & 2359808 \\
\hline Block5_pool (maxPooling2D) & (None, $7,7,512)$ & 0 \\
\hline Flatten (flatten) & (None, 25088) & 0 \\
\hline Fcl (dense) & (None, 4096) & 102764544 \\
\hline
\end{tabular}


TABle 1: Continued.

\begin{tabular}{lcc}
\hline Layer (type) & Output shape & Parameters number \\
\hline Dropout (dropout) & (None, 4096) & 0 \\
Fc2 (dense) & (None, 4096) & 16781312 \\
Dropout_1 (dropout) & (None, 4096) & 0 \\
Fc3 (dense) & (None, 8192) & 33562624 \\
Dropout_2 (dropout) & (None, 8192) & 0 \\
Fc4 (dense) & (None, 8192) & 67117056 \\
Dropout_3 (dropout) & (None, 8192) & 0 \\
Fc5 (dense) & (None, 8192) & 67117056 \\
Dropout_4 (dropout) & (None, 8192) & 0 \\
Fc6 (dense) & (None, 16384) & 134234112 \\
Dropout_5 (dropout) & (None, 16384) & 0 \\
Dense_class_2 (dense) & (None, 2) & 32770 \\
\hline
\end{tabular}

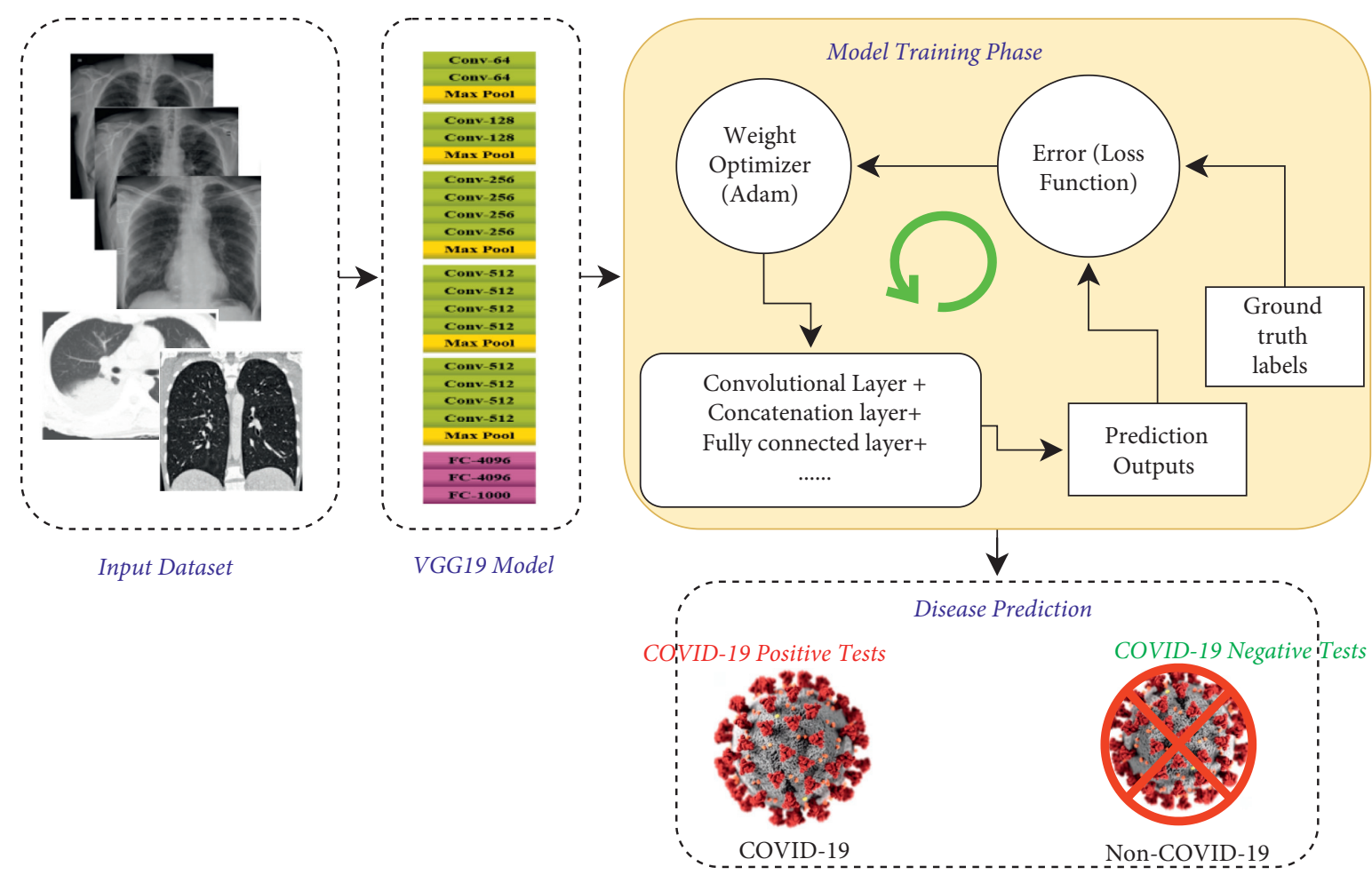

FIgure 3: VGGNet model for COVID-19 diagnosis.

TABle 2: Symptoms dataset.

\begin{tabular}{lcccc}
\hline Patient ID & 1 & 2 & 3 & 270 \\
\hline Male & 0 & 0 & 1 & 0 \\
Female & 1 & 1 & 37 & 1 \\
Age & 28 & 51 & 1 & 25 \\
Fever & 1 & 1 & 0 & 0 \\
Cough & 1 & 1 & 0 & 0 \\
Shortness of breath & 1 & 1 & 0 & 0 \\
Sore throat & 0 & 0 & 0 & 0 \\
Chills & 0 & 0 & 0 & 0 \\
Muscle pain & 0 & 0 & 0 & 0 \\
Nausea & 0 & 1 & 0 & 0 \\
Diarrhea & 0 & 1 & 0 & 0 \\
Fatigue & 1 & 0 & 0 \\
Vomiting & 0 & 0 & 0 \\
Headache & 0 & 0 & 0 \\
Malaise & 1 & 0 & 0 \\
Output (classes) & 1 & 1 & 0 \\
\hline
\end{tabular}


TABLE 3: X-ray and CT datasets.

\begin{tabular}{lcc}
\hline Datasets & COVID-19 & Normal \\
\hline CT images & 349 & 397 \\
X-ray images & 910 & 1341 \\
Combined images & 1259 & 1738 \\
\hline
\end{tabular}

Normal

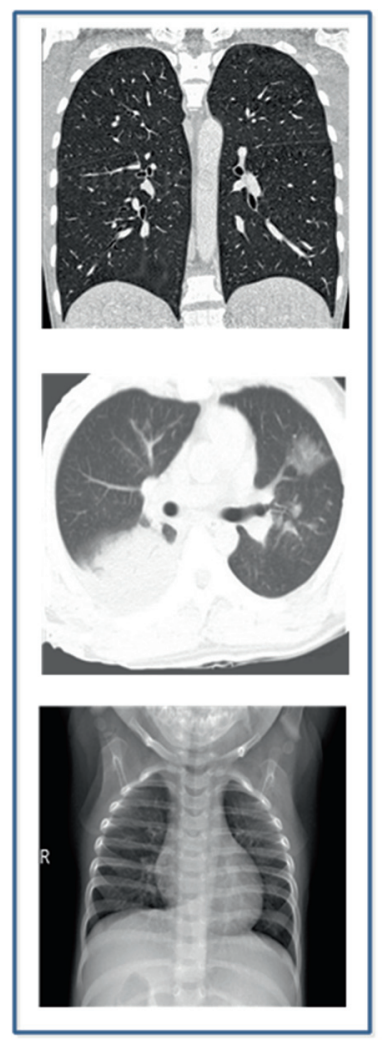

COVID-19

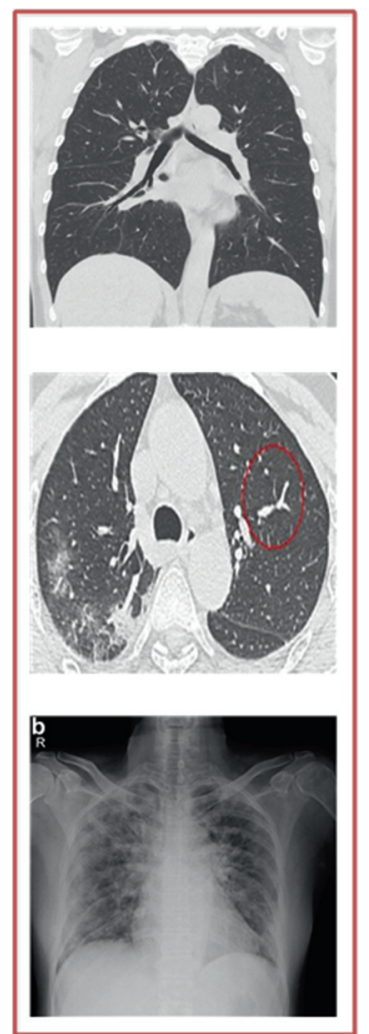

Figure 4: Example of X-ray and CT images of a normal patient and a COVID-19 patient.

4.3. Evaluation Metrics. Regarding the logistic regression (VGG19) prediction (classification) task, specific metrics are denoted as follows: incorrectly predicted (classified) healthy cases (False Negatives, FN), incorrectly predicted (classified) diseased cases (False Positives, FP), correctly identified healthy cases (True Negatives, TN), and correctly identified diseased cases (True Positives, TP). Based on these metrics, we use equations (1) to (4) to calculate the accuracy, precision, recall, and F1-score, respectively.

$$
\begin{aligned}
\text { Accuracy } & =\frac{\mathrm{TP}+\mathrm{TN}}{\mathrm{TP}+\mathrm{FP}+\mathrm{FN}+\mathrm{TN}}, \\
\text { Precision } & =\frac{\mathrm{TP}}{\mathrm{TP}+\mathrm{FP}}, \\
\text { Recall } & =\frac{\mathrm{TP}}{\mathrm{TP}+\mathrm{FN}}, \\
\text { F1_score } & =2 \times \frac{\text { Recall } \times \text { Precision }}{\text { Recall }+ \text { Precision }} .
\end{aligned}
$$

\section{Numerical Results}

The experimental results have been introduced in this section to evaluate our suggested framework based on ML/ DL techniques. We begin by evaluating the symptoms checker model by selecting the most effective ML technique. Thereafter, we evaluate the performance of the proposed COVID-19 diagnosis model by exploiting CT images, then using X-ray images, and finally by using both in the same dataset.

5.1. Regression Technique-Based Symptoms Checker Model. To select one of the suggested algorithms as the brain for our symptoms checker model, we implement different ML techniques (KNN, SVM, and logistic regression) and then evaluate their performance metrics. Here, we consider the explained dataset, consisting of the patient's ID and symptoms input, where the output is labeled " 0 " to indicate that the patient is not infected (low infection probability $\leq 40 \%$ ) and " 1 " to indicate that the patient is infected (high infection probability $\geq 50 \%$ ). Table 4 further clarifies the dataset.

Table 4 shows that the logistic regression model used to check the subject's symptoms outperforms the other ML algorithms regarding their best performance in terms of accuracy, precision, recall, and F1-score. Overall, the logistic regression model achieves an average accuracy classification of $51.18 \%$ in two classes (COVID-19 and normal) compared with SVM and KNN, which achieved accuracy scores of 0.481 and 0.444 , respectively. Regarding the other evaluation metrics, the logistic regression model reached the highest mean values for all classes. Based on these results, we consider the logistic regression technique as the brain tool to predict infection probability for patients.

To assess the framework's symptoms checker, we created a test application that provides a small graphical interface for selecting symptoms and obtaining the predicted infection probability. Figure 5 represents the graphical interface, created using Python, which contains symptom buttons; the doctor needs only to select the symptoms, and then the application will predict the infection probability of this patient (a positive case in the figure example). This interface also provides the total number of cases in the world from the website of the World Health Organization (WHO). Some advice will be provided when the symptoms test shows positive results. Note that this application also saves the patients' test results to be used as historical data to predict other cases and to increase the prediction accuracy.

5.2. VGGNet Technique-Based COVID-19 Diagnosis Model. The proposed deep learning model was trained and tested using the Python programming language [39]. The Adam optimizer technique is a powerful technique that updates the weights at each iteration and minimizes the gradient error between the ground truth labels and the prediction outputs [40]. In addition, the overall experiments were implemented on an Intel Core TM i7-3770@3.4 GHz CPU 
TABle 4: Performance evaluation for machine learning models.

\begin{tabular}{|c|c|c|c|c|c|c|}
\hline ML model & Accuracy score & Class & Precision & Recall & F1-score & Support \\
\hline \multirow{2}{*}{$\begin{array}{l}\text { Logistic } \\
\text { regression }\end{array}$} & \multirow{2}{*}{0.518} & 0 & 0.86 & 0.33 & 0.48 & 18 \\
\hline & & 1 & 0.40 & 0.89 & 0.55 & 9 \\
\hline \multirow{2}{*}{ KNN } & \multirow{2}{*}{0.444} & 0 & 0.67 & 0.33 & 0.44 & 18 \\
\hline & & 1 & 0.33 & 0.67 & 0.44 & 9 \\
\hline \multirow{2}{*}{ SVM } & \multirow{2}{*}{0.481} & 0 & 0.75 & 0.33 & 0.46 & 18 \\
\hline & & 1 & 0.37 & 0.78 & 0.50 & 9 \\
\hline
\end{tabular}

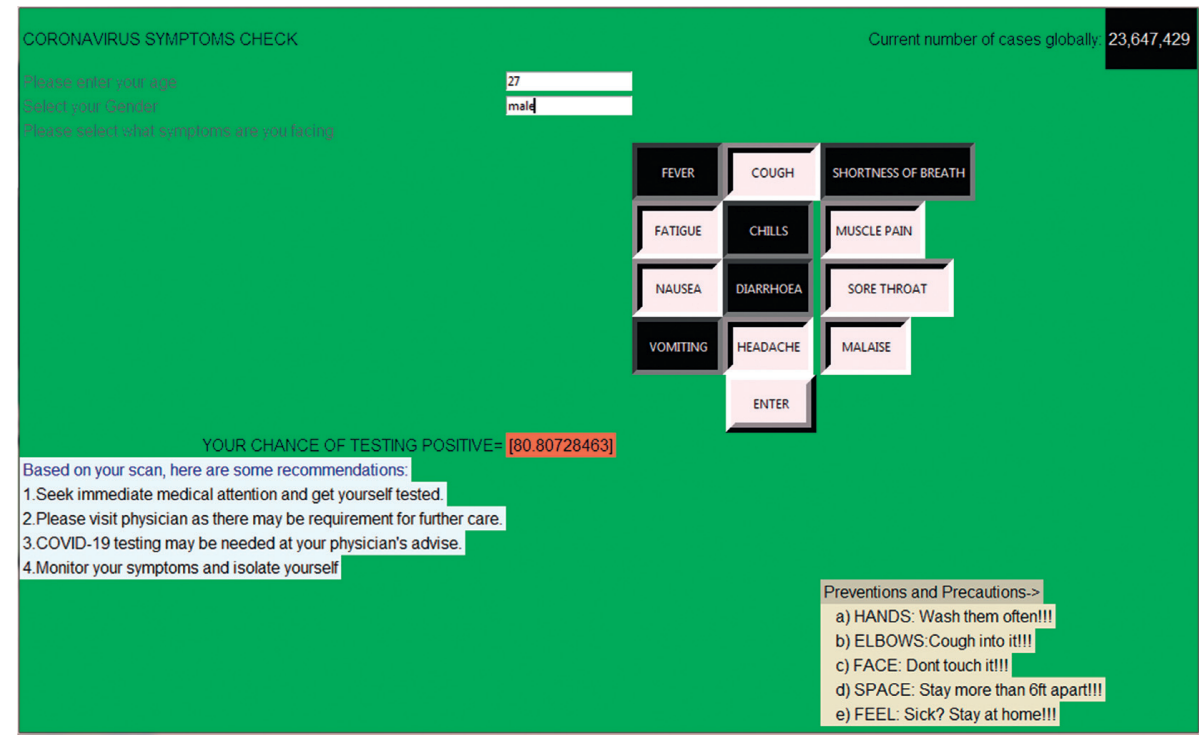

FIGURE 5: PSC application.

and 16 GB RAM. We also use the NVIDIA GeForce RTX 2070 GPU to improve the speed of the proposed trained deep model. The deep learning parameters applied in this work are as follows. The number of epochs, the learning rate, and the batch size were experimentally set at 100 , 0.0001 and 64, respectively. The datasets are gathered into two subsets, $80 \%$ for training and $20 \%$ for testing, including normal and COVID-19 cases.

During the training process, as shown in Figure 3, the cross-entropy is the loss function $(L)$, mentioned in equation (6), used in this study, where $g$ represents the ground truth labels and $p$ denotes the prediction outputs.

$L(g, p)=\frac{1}{M} \sum_{i=0}^{M}\left(g * \log \left(p_{i}\right)+(1-g) * \log \left(1-p_{i}\right)\right)$.

The training performances in terms of the loss function, validation loss, accuracy rate, precision, recall, specificity, F1-scores, and validation accuracy for the deep learning model will be introduced for the X-ray dataset, followed by the CT dataset, and finally for the combined datasets.

5.2.1. VGG19 Based on X-Ray Images. In this study, the performances of the proposed pretrained VGG19 model were evaluated using X-ray datasets to detect COVID-19. Table 5 summarizes the performance evaluation of the proposed deep model in terms of precision, recall,
TAble 5: Performance of VGG19 network based on X-ray dataset.

\begin{tabular}{lcc}
\hline Metrics & COVID-19 & Normal \\
\hline Precision & 0.97 & 0.97 \\
Recall & 0.96 & 0.98 \\
Specificity & 0.98 & 0.98 \\
F1-score & 0.97 & 0.98 \\
\hline
\end{tabular}

specificity, and F1-score for the two classes. Based on the experimental findings, the proposed VGG19 classifier achieves a precision of $97 \%$ for the two classes, and the achieved recall values for the COVID-19 and normal classes are $96 \%$ and $98 \%$, respectively. Further, the specificity reaches $98 \%$ for normal and COVID-19 cases, while the F1-scores for the COVID-19 and normal classes are 97\% and 98\%, respectively.

Figure 6 shows the accuracy and loss curves on the training and validation processes to evaluate the performance of the proposed VGG19 network. The achieved training and validation accuracy rates are $97.3 \%$ and $97.1 \%$, respectively. Similarly, the training and validation loss values were found to be 0.08 and 0.07 , respectively, for the proposed model. Consequently, the proposed deep learning model reaches a good performance level of detection. For the testing phase, we tested our trained model to evaluate the VGG19 classifier for the detection of COVID-19 and normal cases on the X-ray datasets, as illustrated in Figure 7. 


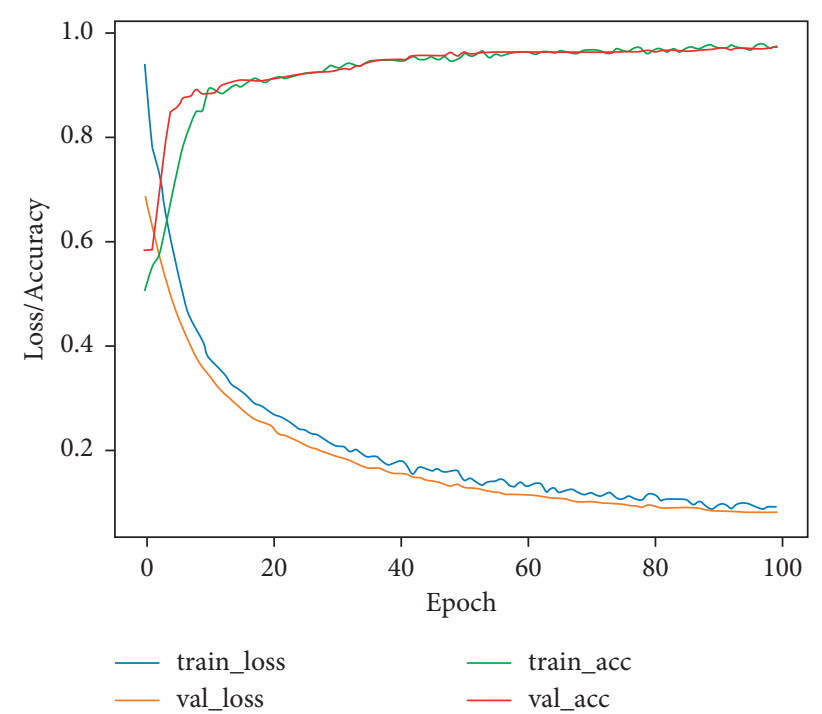

FIgure 6: Accuracy and loss for COVID-19 detection.

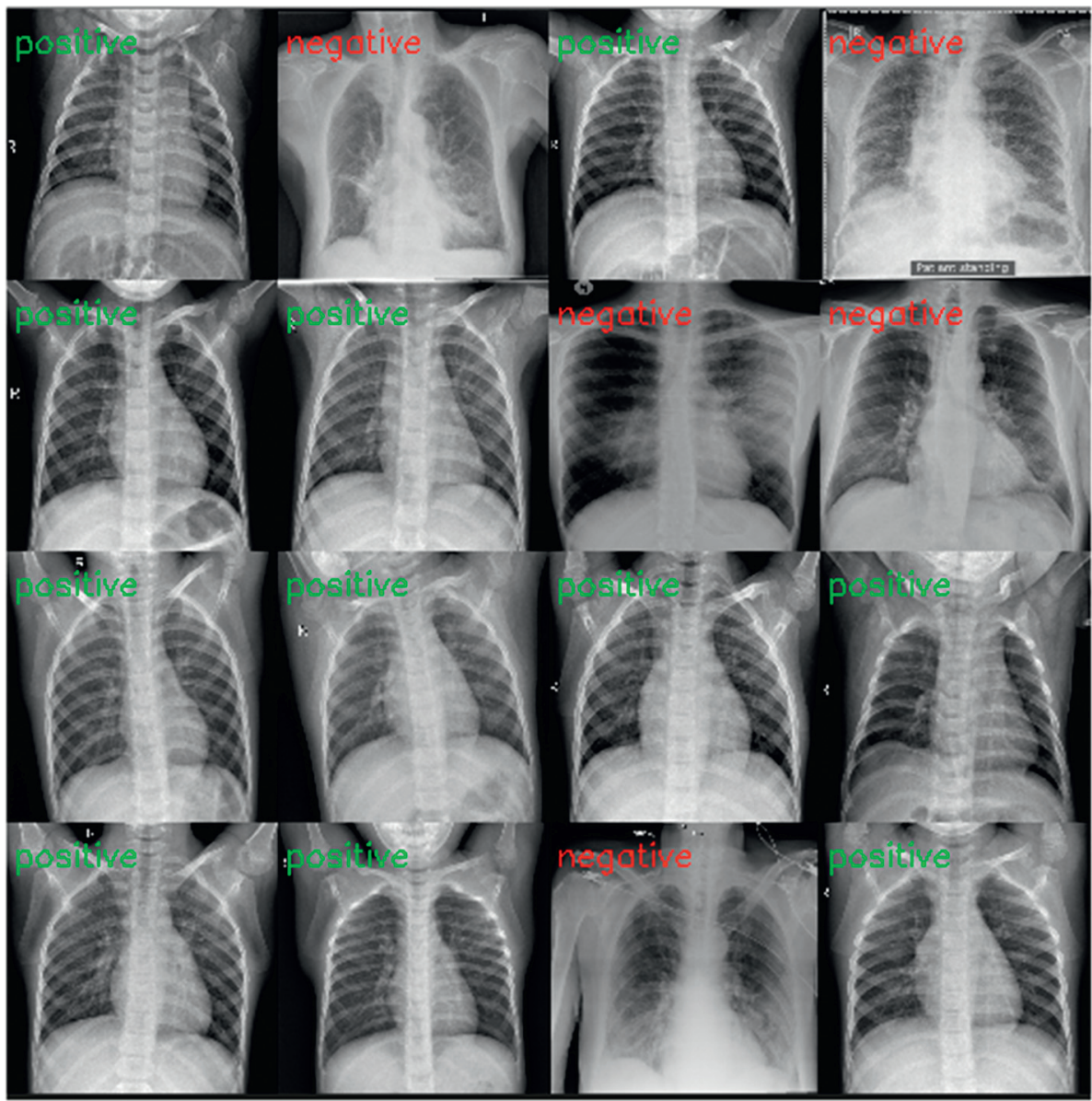

Figure 7: Tested VGG19 model.

5.2.2. VGG19 Based on CT Images. In this study, the performances of the proposed pretrained VGG19 model were evaluated using CT datasets to detect COVID-19. Table 6 summarizes the performance evaluation of the proposed deep model in terms of precision, recall, specificity, and F1score for the two classes. Based on the experimental findings, the proposed VGG19 classifier achieves an accuracy of $86 \%$ for normal and COVID-19 cases. The precision is $73 \%$ and 
TABLE 6: Performance of VGG19 network based on CT dataset.

\begin{tabular}{lcc}
\hline Metrics & COVID-19 & Normal \\
\hline Precision & 0.73 & 0.85 \\
Recall & 0.86 & 0.72 \\
Specificity & 0.72 & 0.72 \\
F1-score & 0.79 & 0.78 \\
\hline
\end{tabular}

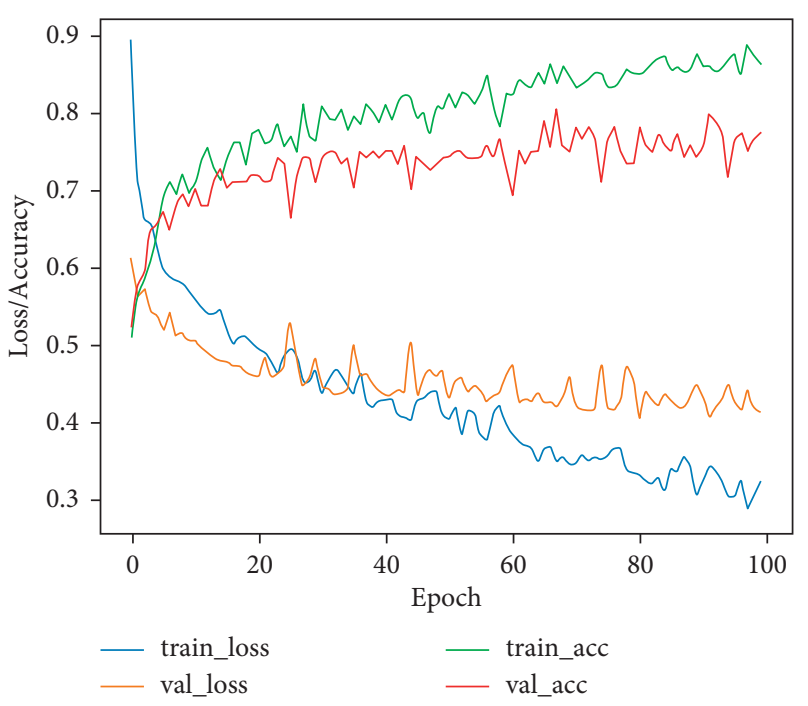

Figure 8: Accuracy and loss for COVID-19 detection.

$85 \%$ for the two classes, and the achieved recall values for the COVID-19 and normal classes are $86 \%$ and $72 \%$, respectively. The specificity is $72 \%$ for both normal and COVID-19 cases, and the F1-scores for the COVID-19 and normal classes are $79 \%$ and $78 \%$, respectively.

Figure 8 shows the accuracy and loss curves on the training and validation processes to evaluate the performance of the proposed VGG19 network. The achieved training and validation accuracy rates are $86 \%$ and $77 \%$, respectively. Similarly, the training and validation loss values are 0.32 and 0.41 , respectively, for the proposed model. For the testing phase, we tested our trained model to evaluate the VGG19 classifier for the detection of COVID-19 and normal cases on the CT dataset, as illustrated in Figure 9.

5.2.3. VGG19 Based on Combined Datasets. In this study, the performances of the proposed pretrained VGG19 model were evaluated using the CT and X-ray datasets, including two classes (normal and COVID-19), to detect COVID-19. Table 7 summarizes the performance evaluation of the proposed deep model in terms of precision, recall, specificity, and F1-score for the two classes. Based on the experimental findings, the proposed VGG19 classifier achieves a precision of $86 \%$ and $91 \%$ for the two classes, and the achieved recall value for both COVID-19 and normal class is $89 \%$. The specificity is $89 \%$ for both normal and COVID-19 cases, and the F1-scores for the COVID-19 and normal classes are $87 \%$ and $90 \%$, respectively.

Figure 10 shows the accuracy and loss curves on the training and validation processes to evaluate the performance of the proposed VGG19 network. The achieved training and validation accuracy rates are $90 \%$ and $88 \%$, respectively. Similarly, the training and validation loss values are 0.20 and 0.21 , respectively, for the proposed model. Consequently, the proposed deep learning model reaches a good performance level of detection. For the testing phase, we tested our trained model to evaluate the VGG19 classifier for the detection of COVID-19 and normal cases on the CT and X-ray datasets, as illustrated in Figure 11.

5.3. Comparative Study. For further evaluation, we have presented the performance comparison of the proposed VGG19 model with the advanced approaches, as shown in Table 8. From this table, we can conclude that the results obtained by our proposed deep learning model for COVID19 detection are slightly superior to those of existing works in terms of evaluated performances. Regarding the accuracy value, our proposed model achieved the best accuracy rates of $97 \%, 86 \%$, and $90 \%$ for normal and COVID-19 classes when using all three datasets-X-ray, CT, and both X-ray and CT, respectively. The authors of [41] developed a deep learning model-namely, COVID-Net-to detect COVID19 and normal cases from X-ray images, and this model achieved an accuracy of $83.5 \%$. Meanwhile, the authors of [42] proposed a CoroNet network for the detection of COVID-19 cases from X-ray images with an accuracy value of around $89.6 \%$. Likewise, a VGG19 and DenseNet201 deep learning system achieved an accuracy value of $90 \%$ using X-ray datasets to classify COVID-19 and normal patients [43]. Moreover, to detect the two cases, the authors of [44] 


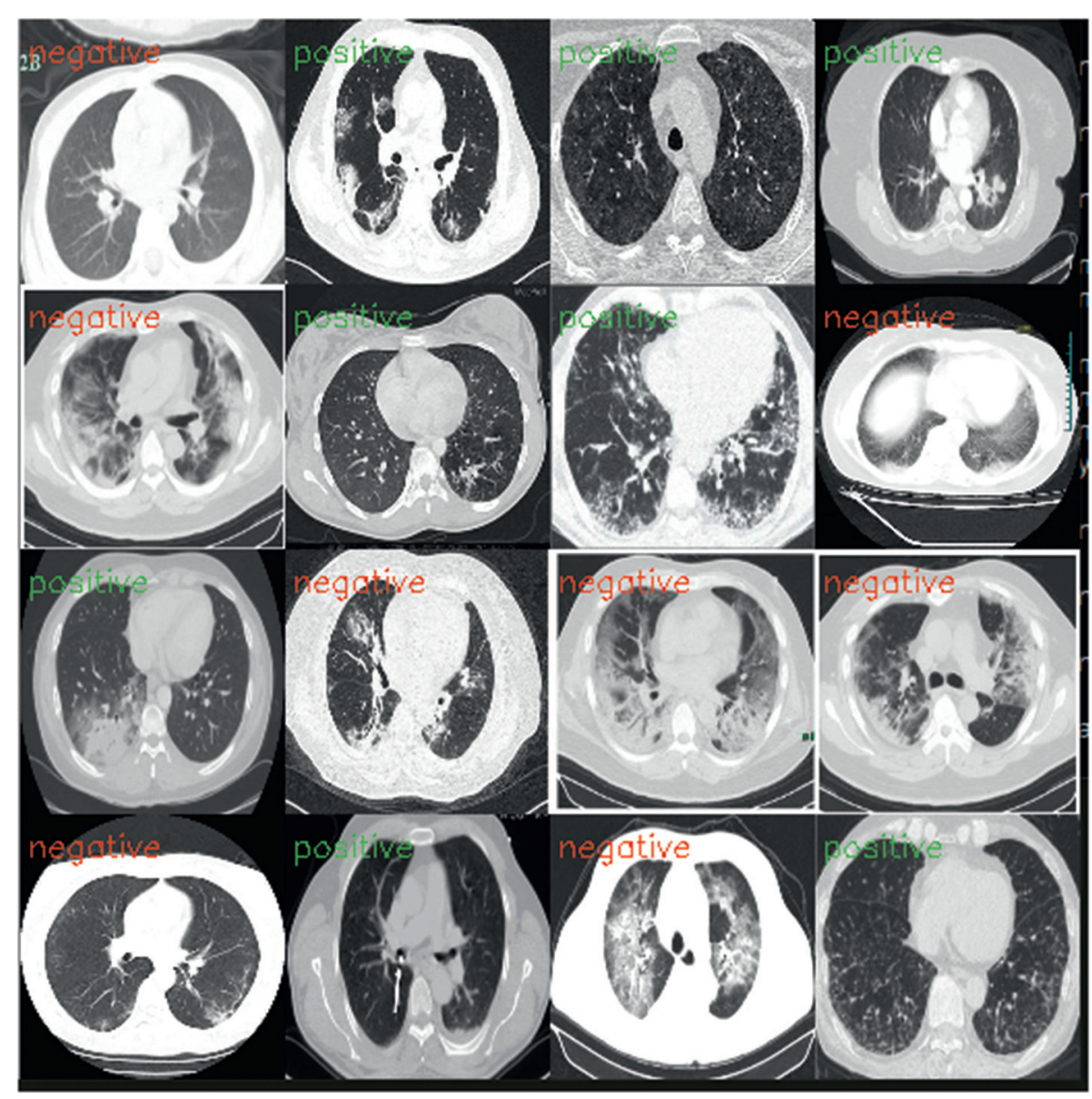

FIgUre 9: Tested VGG19 model.

TABle 7: Performance of VGG19 network based on combined dataset.

\begin{tabular}{lcc}
\hline Metrics & COVID-19 & Normal \\
\hline Precision & 0.86 & 0.91 \\
Recall & 0.89 & 0.89 \\
Specificity & 0.89 & 0.89 \\
F1-score & 0.87 & 0.90 \\
\hline
\end{tabular}

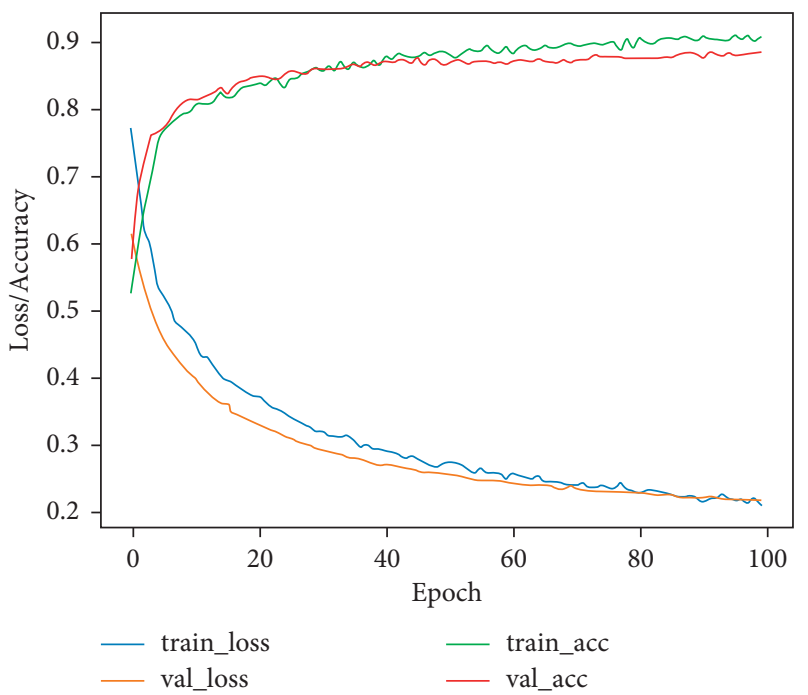

Figure 10: Accuracy and loss for COVID-19 detection. 


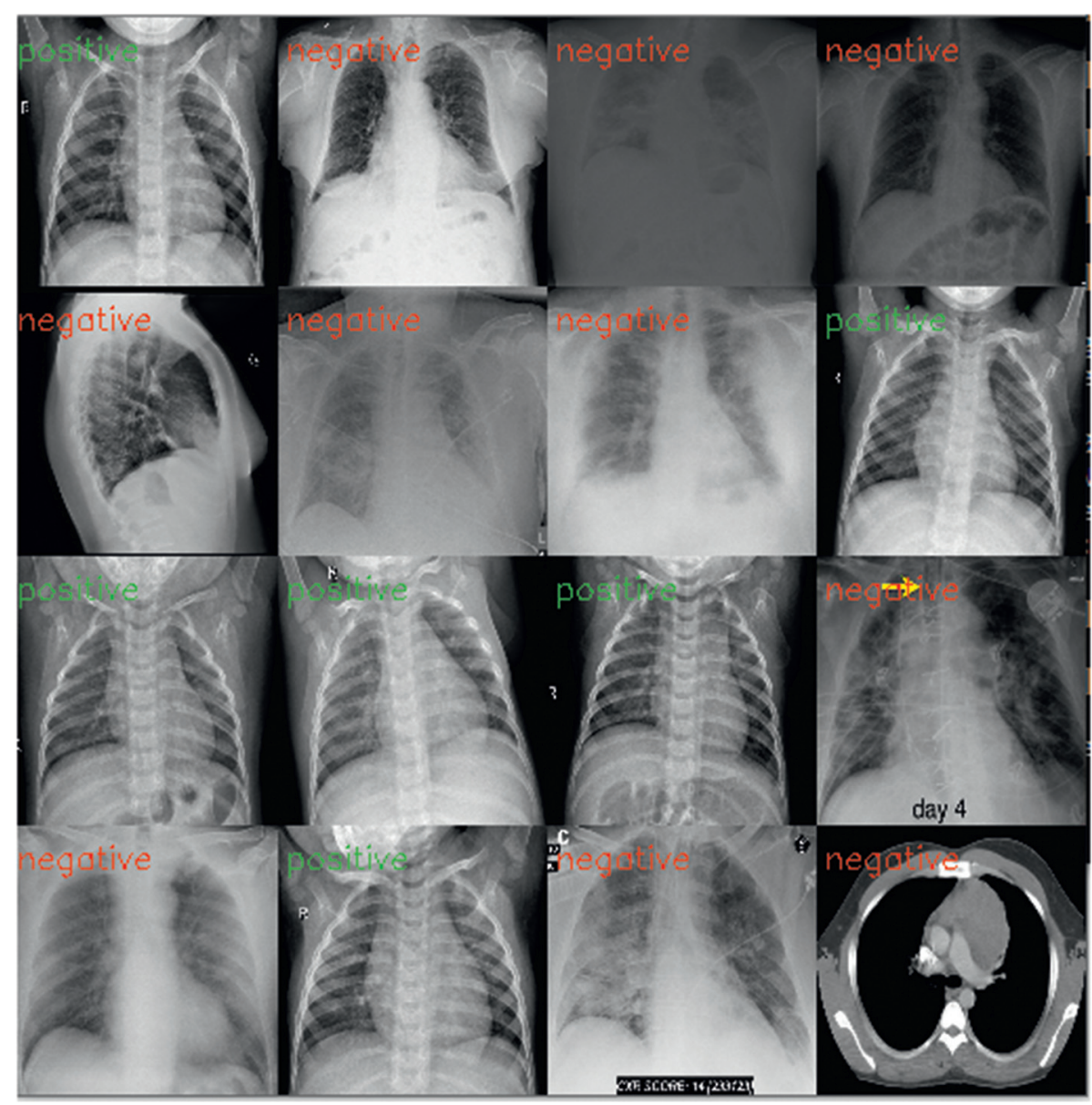

FIGURE 11: Tested VGG19 model.

TABLE 8: Performance comparison of the proposed VGG19 model with the state-of-the-art approaches.

\begin{tabular}{|c|c|c|c|c|c|c|c|}
\hline Approaches & Model & Datasets & Class & Precision & Recall & F1-score & Accuracy \\
\hline [41] & COVID-Net & $\mathrm{X}$-ray & $\begin{array}{c}\text { COVID-19 } \\
\text { Normal }\end{array}$ & $\begin{array}{c}80 \\
95.1\end{array}$ & $\begin{array}{l}100 \\
73.9\end{array}$ & $\begin{array}{c}88.8 \\
83.17\end{array}$ & 83.5 \\
\hline [42] & CoroNet & X-ray & $\begin{array}{c}\text { COVID-19 } \\
\text { Normal }\end{array}$ & $\begin{array}{l}93.17 \\
95.25\end{array}$ & $\begin{array}{c}98.25 \\
93.5\end{array}$ & $\begin{array}{c}95.61 \\
94.3\end{array}$ & 89.6 \\
\hline [43] & $\begin{array}{c}\text { VGG19+ } \\
\text { DenseNet201 }\end{array}$ & $\mathrm{X}$-ray & $\begin{array}{c}\text { COVID-19 } \\
\text { Normal }\end{array}$ & $\begin{array}{c}83 \\
100\end{array}$ & $\begin{array}{c}100 \\
80\end{array}$ & $\begin{array}{l}91 \\
89\end{array}$ & 90 \\
\hline [44] & $\mathrm{CNN}$ & CT & $\begin{array}{l}\text { COVID-19 } \\
\text { Normal }\end{array}$ & 81.73 & 85 & 83.33 & 83 \\
\hline [45] & $\begin{array}{c}\text { RF } \\
\text { GBM } \\
\text { KNN }\end{array}$ & $\begin{array}{l}\text { X-ray } \\
\text { X-ray } \\
\text { X-ray }\end{array}$ & $\begin{array}{l}- \\
- \\
-\end{array}$ & $\begin{array}{l}96 \\
93 \\
99\end{array}$ & $\begin{array}{l}- \\
-\end{array}$ & $\begin{array}{l}95 \\
92 \\
93\end{array}$ & $\begin{array}{l}95 \\
92 \\
93\end{array}$ \\
\hline [46] & EfficientNet-B4+CLAHE & $\mathrm{CT}$ & - & 86.81 & 78.27 & 82.32 & 83.43 \\
\hline Proposed & VGG19 & $\begin{array}{c}\text { X-ray } \\
\text { CT + X-ray }\end{array}$ & $\begin{array}{c}\text { COVID-19 } \\
\text { Normal } \\
\text { COVID-19 } \\
\text { Normal } \\
\text { COVID-19 } \\
\text { Normal }\end{array}$ & $\begin{array}{l}73 \\
85 \\
97 \\
97 \\
86 \\
91\end{array}$ & $\begin{array}{l}86 \\
72 \\
96 \\
98 \\
89 \\
89\end{array}$ & $\begin{array}{l}79 \\
78 \\
97 \\
98 \\
87 \\
90\end{array}$ & 90 \\
\hline
\end{tabular}

proposed a CNN architecture based on CT images which achieved $83 \%$ accuracy. We also compared our experiments with some relevant ML models, such as random forest (RF) (accuracy of about 95\%), gradient boosting machine (GBM) (accuracy of about 92\%), and KNN (accuracy of about 93\%), proposed by [45]. In addition, the proposed technique was 
compared to the EfficientNet-B4 architecture, which uses a three-layer artificial neural network with an accuracy of about $83.43 \%$, probably as a result of using fewer layers compared to our proposed scheme.

In summary, all the results of the experiments reveal that the proposed technique obtains good results in detecting COVID-19 cases compared with other related approaches. This comparative analysis and comparing the overall performance results achieved by the proposed deep CNN model provide strong evidence of the applicability of the model in COVID-19 diagnosis using X-ray and CT image classification.

\section{Conclusion}

SARS-CoV-2 is part of a large family of viruses that cause illnesses ranging from mild colds to fatal diseases. Thanks to advanced AI technology, the early detection of this virus will aid in the rapid recovery of COVID-19 patients. This paper proposed an innovative healthcare center that will be implemented in the cloud and gives access to doctors to diagnose and detect COVID-19 infection. We also propose a PSC based on a regression model to provide initial medical screening for patients and predict whether the patient is infected based on their symptoms. Therefore, based on the pretrained deep learning classifier VGG19, we propose a learning model to classify normal and COVID-19 cases using a dataset of X-ray and CT images. The performance results prove that the proposed scheme yields the highest accuracy of $90 \%$ compared with the others. We seek, in the near future, to add more classification classes such as bacterial pneumonia and other types of viral pneumonia.

\section{Data Availability}

The data used to support the findings of this study are available from the corresponding author upon request.

\section{Conflicts of Interest}

The authors declare that they have no conflicts of interest.

\section{References}

[1] D. S. Hui, E. Azhar, T. A. Madani et al., "The continuing 2019nCoV epidemic threat of novel coronaviruses to global healththe latest 2019 novel coronavirus outbreak in Wuhan, China," International Journal of Infectious Diseases, vol. 91, pp. 264-266, 2020.

[2] M. Shorfuzzaman, M. S. Hossain, and M. F. Alhamid, "Towards the sustainable development of smart cities through mass video surveillance: a response to the COVID-19 pandemic," Sustainable Cities and Society, vol. 64, Article ID 102582, 2021.

[3] Z. Xu, L. Shi, Y. Wang et al., "Pathological findings of COVID19 associated with acute respiratory distress syndrome," The Lancet Respiratory Medicine, vol. 8, no. 4, pp. 420-422, 2020.

[4] Y. Liu, G. Liu, and Q. Zhang, "Deep learning and medical diagnosis," The Lancet, vol. 394, no. 10210, pp. 1709-1710, 2019.
[5] M. Loey, F. Smarandache, and N. E. M Khalifa, "Within the lack of chest COVID-19 X-ray dataset: a novel detection model based on GAN and deep transfer learning," Symmetry, vol. 12, no. 4, Article ID 651, 2020.

[6] I. D. Apostolopoulos and T. A. Mpesiana, "Covid-19: automatic detection from X-ray images utilizing transfer learning with convolutional neural networks," Physical and Engineering Sciences in Medicine, vol. 43, no. 2, pp. 635-640, 2020.

[7] L. Khriji, A. Ammari, S. Messaoud, S. Bouaafia, A. Maraoui, and M. Machhout, "COVID-19 recognition based on patient's coughing and breathing patterns analysis: deep learning approach," in Proceedings of the 2021 29th Conference of Open Innovations Association (FRUCT), pp. 185-191, Tampere, Finland, May 2021.

[8] X. Xiaowei, J. Xiangao, and M. Chunlian, "A deep learning system to screen novel coronavirus disease 2019 pneumonia," Engineering, vol. 6, no. 10, pp. 1122-1129, 2020.

[9] W. Shuai, K. Bo, M. Jinlu et al., "A deep learning algorithm using CT images to screen for corona virus disease (COVID19)," European Radiology, vol. 31, no. 8, pp. 1-9, 2021.

[10] J. Chen, L. Wu, J. Zhang et al., "Deep learning-based model for detecting 2019 novel coronavirus pneumonia on high-resolution computed tomography: a prospective study," Scientific Reports, vol. 10, no. 1, pp. 1-11, 2020.

[11] Y. Bai, L. Yao, T. Wei et al., "Presumed asymptomatic carrier transmission of COVID-19," JAMA, vol. 323, no. 14, pp. 1406-1407, 2020.

[12] H. Chen, J. Guo, C. Wang et al., "Clinical characteristics and intrauterine vertical transmission potential of COVID-19 infection in nine pregnant women: a retrospective review of medical records," The Lancet, vol. 395, no. 10226, pp. 809-815, 2020.

[13] W. Li, M. J. Moore, N. Vasilieva et al., “Angiotensin-converting enzyme 2 is a functional receptor for the SARS coronavirus," Nature, vol. 426, no. 6965, pp. 450-454, 2003.

[14] E. I. Azhar, S. A. El-Kafrawy, S. A. Farraj et al., "Evidence for camel-to-human transmission of MERS coronavirus," New England Journal of Medicine, vol. 370, no. 26, pp. 2499-2505, 2014.

[15] V. M. Corman, O. Landt, M. Kaiser et al., "Detection of 2019 novel coronavirus (2019-nCoV) by real-time RT-PCR," European communicable disease bulletin, vol. 25, no. 3, Article ID 2000045, 2020.

[16] L. Lan, D. Xu, G. Ye et al., "Positive RT-PCR test results in patients recovered from COVID-19," JAMA, vol. 323, no. 15, pp. 1502-1503, 2020.

[17] W. H. Self, D. M. Courtney, C. D. McNaughton, R. G. Wunderink, and J. A. Kline, "High discordance of chest $\mathrm{X}$-ray and computed tomography for detection of pulmonary opacities in ED patients: implications for diagnosing pneumonia," The American Journal of Emergency Medicine, vol. 31, no. 2, pp. 401-405, 2013.

[18] H. C. Magree, F. M. Russell, R. Sa'aga et al., "Chest X-rayconfirmed pneumonia in children in fiji," Bulletin of the World Health Organization, vol. 83, pp. 427-433, 2005.

[19] A. Jacobi, M. Chung, A. Bernheim, and C. Eber, "Portable chest X-ray in coronavirus disease-19 (COVID-19): a pictorial review," Clinical Imaging, vol. 64, pp. 35-42, 2020.

[20] H. Y. F. Wong, H. Y. S. Lam, A. H. T. Fong et al., "Frequency and distribution of chest radiographic findings in COVID-19 positive patients," Radiology, vol. 296, Article ID 201160, 2020.

[21] A. Borghesi and R. Maroldi, "COVID-19 outbreak in Italy: experimental chest $\mathrm{X}$-ray scoring system for quantifying and 
monitoring disease progression," La Radiologia Medica, vol. 125, pp. 509-513, 2020.

[22] X. Wang, Y. Peng, L. Lu, Z. Lu, M. Bagheri, and R. M. Summers, "Chestx-ray8: hospital-scale chest X-ray database and benchmarks on weakly-supervised classification and localization of common thorax diseases," in Proceedings of the IEEE Conference on Computer Vision and Pattern Recognition, pp. 2097-2106, Honolulu, HI, USA, July 2017.

[23] P. Rajpurkar, J. Irvin, K. Zhu et al., "Chexnet: radiologist-level pneumonia detection on chest X-rays with deep learning," 2017, https://arxiv.org/abs/1711.05225.

[24] H. Wang, H. Jia, L. Lu, and Y. Xia, "Thorax-Net: an attention regularized deep neural network for classification of thoracic diseases on chest radiography," IEEE Journal of Biomedical and Health Informatics, vol. 24, no. 2, pp. 475-485, 2019.

[25] S. Bouaafia, S. Messaoud, R. Khemiri, and F. Elzahra Sayadi, "COVID-19 recognition based on deep transfer learning," in Proceedings of the 2021 IEEE International Conference on Design \& Test of Integrated Micro \& Nano-Systems (DTS), Sfax, Tunisia, June 2021.

[26] D. S. Kermany, M. Goldbaum, W. Cai et al., "Identifying medical diagnoses and treatable diseases by image-based deep learning," Cell, vol. 172, no. 5, pp. 1122-1131, 2018.

[27] S. Rajaraman, S. Candemir, I. Kim, G. Thoma, and S. Antani, "Visualization and interpretation of convolutional neural network predictions in detecting pneumonia in pediatric chest radiographs," Applied Sciences, vol. 8, no. 10, Article ID 1715, 2018.

[28] M. Kim, C. Yan, D. Yang, Q. Wang, J. Ma, and G. Wu, Deep learning in biomedical image analysis, Biomedical Information Technology, Academic Press, Cambridge, MA, USA, pp. 239-263, 2020.

[29] M. Eliezer, C. Hautefort, A. L. Hamel et al., "Sudden and complete olfactory loss function as a possible symptom of COVID-19," JAMA Otolaryngology-Head and Neck Surgery, vol. 146, 2020.

[30] B. Venkataramanaiah and J. Kamala, "ECG signal processing and KNN classifier-based abnormality detection by VHdoctor for remote cardiac healthcare monitoring," Soft Computing, vol. 24, pp. 1-10, 2020.

[31] P. Khanna and S. Kumar, "Malaria parasite classification employing chan-vese algorithm and SVM for healthcare," in Proceedings of the First International Conference on Computing, Communications, and Cyber-Security (IC4S 2019), Singapore, April 2020.

[32] C.-Y. J. Peng, K. L. Lee, and G. M. Ingersoll, "An introduction to logistic regression analysis and reporting," The Journal of Educational Research, vol. 96, no. 1, pp. 3-14, 2002.

[33] A. Di Crescenzo and P. Paraggio, "Logistic growth described by birth-death and diffusion processes," Mathematics, vol. 7, no. 6, Article ID 489, 2019.

[34] K. Simonyan and A. Zisserman, "Very deep convolutional networks for large-scale image recognition," 2014, https:// arxiv.org/abs/1409.1556.

[35] Y. Zoabi, S. Deri-Rozov, and N. Shomron, "Machine learningbased prediction of COVID-19 diagnosis based on symptoms," Npj Digital Medicine, vol. 4, no. 1, pp. 3-5, 2021.

[36] CT dataset source: https://github.com/UCSD-AI4H/COVIDCT.

[37] https://www.kaggle.com/sudalairajkumar/novel-corona-virus2019-dataset/data.

[38] https://github.com/rekalantar/covid19_detector/tree/master/ dataset.
[39] A. Gulli, A. Kapoor, and S. Pal, Deep Learning with TensorFlow 2 and Keras: Regression, ConvNets, GANs, RNNs, NLP, and More with TensorFlow 2 and the Keras API, Packt Publishing, Limited, Birmingham, UK, 2019.

[40] D. P. Kingma and J. Ba, "Adam: a method for stochastic optimization," 2014, https://arxiv.org/abs/1412.6980.

[41] L. Wang and A. Wong, "COVID-Net: a tailored deep convolutional neural network design for detection of COVID-19 cases from chest radiography images," 2020, https://arxiv.org/ abs/2003.09871.

[42] A. I. Khan, J. L. Shah, and M. M. Bhat, "Coronet: a deep neural network for detection and diagnosis of COVID-19 from chest x-ray images," Computer Methods and Programs in Biomedicine, vol. 196, Article ID 105581, 2020.

[43] E. E. D. Hemdan, M. A. Shouman, and M. E. Karar, "Covidxnet: a framework of deep learning classifiers to diagnose COVID-19 in x-ray images," 2020, https://arxiv.org/abs/2003. 11055.

[44] M. Polsinelli, L. Cinque, and G. Placidi, "A Light CNN for detecting COVID-19 from CT scans of the chest," 2020, https://arxiv.org/abs/2004.12837.

[45] A. A. Reshi, F. Rustam, A. Mehmood et al., "An efficient CNN model for COVID-19 disease detection based on X-ray image classification," Complexity, vol. 2021, Article ID 6621607, 12 pages, 2021.

[46] G. Marques, D. Agarwal, and I. de la Torre Díez, “Automated medical diagnosis of COVID-19 through EfficientNet convolutional neural network," Applied Soft Computing, vol. 96, Article ID 106691, 2020. 